\title{
Managing the solvent water polarization to obtain improved NMR spectra of large molecular structures
}

\section{Journal Article}

Author(s):

Hiller, Sebastian; Wider, Gerhard; Etezady-Esfarjani, Touraj; Horst, Reto; Wüthrich, Kurt

Publication date:

2005-05

Permanent link:

https://doi.org/10.3929/ethz-b-000032117

Rights / license:

In Copyright - Non-Commercial Use Permitted

Originally published in:

Journal of Biomolecular NMR 32(1), https://doi.org/10.1007/s10858-005-3070-8 


\title{
Managing the solvent water polarization to obtain improved NMR spectra of large molecular structures
}

\author{
Sebastian Hiller, Gerhard Wider*, Touraj Etezady-Esfarjani, Reto Horst** \& Kurt \\ Wüthrich \\ Institut für Molekularbiologie und Biophysik, Eidgenössische Technische Hochschule Zürich, CH-8093, \\ Zürich, Switzerland
}

Received 20 December 2004; Accepted 17 February 2005

Key words: CRIPT, paramagnetic relaxation enhancement, sensitivity, spin diffusion, TROSY, water suppression

\begin{abstract}
In large molecular structures, the magnetization of all hydrogen atoms in the solute is strongly coupled to the water magnetization through chemical exchange between solvent water and labile protons of macromolecular components, and through dipole-dipole interactions and the associated "spin diffusion" due to slow molecular tumbling. In NMR experiments with such systems, the extent of the water polarization is thus of utmost importance. This paper presents a formalism that describes the propagation of the water polarization during the course of different NMR experiments, and then compares the results of model calculations for optimized water polarization with experimental data. It thus demonstrates that NMR spectra of large molecular structures can be improved with the use of paramagnetic spin relaxation agents which selectively enhance the relaxation of water protons, so that a substantial gain in signal-to-noise can be achieved. The presently proposed use of a relaxation agent can also replace the water flip-back pulses when working with structures larger than about $30 \mathrm{kDa}$. This may be a valid alternative in situations where flip-back pulses are difficult to introduce into the overall experimental scheme, or where they would interfere with other requirements of the NMR experiment.
\end{abstract}

Abbreviations: CRIPT - cross relaxation-induced polarization transfer; DOTA - 1, 4, 7, 10-tetra-acetic acid-1, 4, 7, 10-tetraazacyclododecane; TROSY - transverse relaxation-optimized spectroscopy.

\section{Introduction}

During the last decades, NMR structure determination and various NMR applications for the biophysical characterization of proteins and nucleic acids with molecular weights up to about $30 \mathrm{kDa}$ have become standard procedures

\footnotetext{
*To whom correspondence should be addressed. E-mail: gsw@mol.biol.ethz.ch

**Present address: The Scripps Research Institute, La Jolla, CA 92037
}

(Wüthrich, 1986; Bax and Grzesiek, 1993; Kay, 1997; Wider, 1998; Wüthrich, 2003). For structures of higher molecular weight, NMR studies are more difficult, mainly because of fast transverse spin relaxation due to slow molecular tumbling (Brownian motion). In the last few years, NMR techniques for large molecules have been developed, which improve the quality of the spectra through optimization of transverse relaxation during evolution and acquisition times (TROSY) (Pervushin et al., 1997; Wüthrich and Wider, 2003), and through the use of both cross 
correlated relaxation-induced polarization transfer and relaxation optimization during magnetization transfer steps (CRINEPT) (Riek et al., 1999) in multi-dimensional NMR experiments. Combined with perdeuteration of the non-labile proton positions of the macromolecule (LeMaster, 1994), these techniques have so far enabled studies with molecular sizes up to $870 \mathrm{kDa}$ (Fiaux et al., 2002).

In typical NMR samples of proteins in aqueous solution, the water concentration is about 50,000 -fold higher than the concentration of the macromolecule, and the correspondingly large intensity of the water resonance needs special consideration in NMR experiments. A common method of dealing with the solvent magnetization is presaturation of the water resonance (Wider et al., 1983). Since water protons undergo chemical exchange with labile protons of proteins and nucleic acids, i.e., oxygen- or nitrogen-bound protons that are not involved in hydrogen bonds, saturation of the water resonance leads to simultaneous saturation of these protons (Grzesiek and Bax, 1993; Wang et al., 1996). In large molecular structures, spin diffusion efficiently transfers the saturation from labile protons further to non-exchangeable hydrogen atoms, thus reducing their signal intensities and leading to a general deterioration of the NMR spectra (Akasaka et al., 1978; Stoesz et al., 1978). This general signal loss can be minimized by maintaining a high steady-state water polarization throughout the experiment, which is usually achieved using selective "flip-back" pulses on the water resonance (Grzesiek and Bax, 1993). Similar effects have been observed between the aliphatic proton resonances and the remainder of the spectrum in fully protonated large molecular systems (Akasaka et al., 1978), and it has been demonstrated that pulse sequences avoiding saturation of the covalently bound aliphatic protons yield more signal (Pervushin et al., 2002).

The presently available water flip-back techniques would have improved efficiency if the longitudinal relaxation time $T_{1}$ of the solvent water protons could be substantially reduced without significantly affecting the relaxation times of the nuclei in the macromolecular solute (Otting, 1997). This paper investigates the application of selective relaxation agents to maintain a large water polarization, and a formalism describing the propagation of water polarization during the course of different NMR experiments is presented and experimentally validated. The increase of the signal intensity in very large macromolecular solutes by adding a suitable concentration of the relaxation agent $\mathrm{Gd}(\mathrm{DOTA})$ (Chang et al., 1993) is demonstrated with the $800 \mathrm{kDa}$ chaperonin GroEL (Xu et al., 1997). We further propose that experiments for studies of solutes with molecular weights larger than about $30 \mathrm{kDa}$, which would normally be recorded with water flip-back pulses, can in the presence of suitably selected relaxation enhancement reagents be performed with comparable sensitivity using saturation of the water resonance.

\section{Theory}

\section{Propagation of the water polarization}

This section presents a formalism to describe the course of the water proton polarization, $M_{z}$, during a typical NMR experiment consisting of a large number of consecutively recorded scans (Figure 1). Each scan consists of a sequence of radio-frequency (rf) pulses, delays between these pulses, an acquisition period during which the free induction decay (FID) is recorded, and a recovery period. The water polarizations before and after the pulses of the $i$ th scan, $M_{z}^{\mathrm{b}, i}$ and $M_{z}^{\mathrm{a}, i}$, are the quantities of interest. If care is taken that the sequence of rf-pulses maintains the water polarization, a certain fraction thereof is left after the pulse sequence,

$$
M_{z}^{\mathrm{a}, i}=f \cdot M_{z}^{\mathrm{b}, i},
$$

wherethe value of the "flip-back ratio", $f$, depends on the quality of the water flip-back pulses and is assumed to have a constant value for a given experimental setup. For pulse sequences which do not invert the water polarization, only values of $f$ in the range $0 \leq f \leq 1$ are possible. A short sequence with a small number of flip-back pulses usually results in higher values of $f$ than a long sequence with many such pulses. In experiments with saturation of the water resonance during the pulse sequence, for example, by application of a strong magnetic field gradient on the transverse 


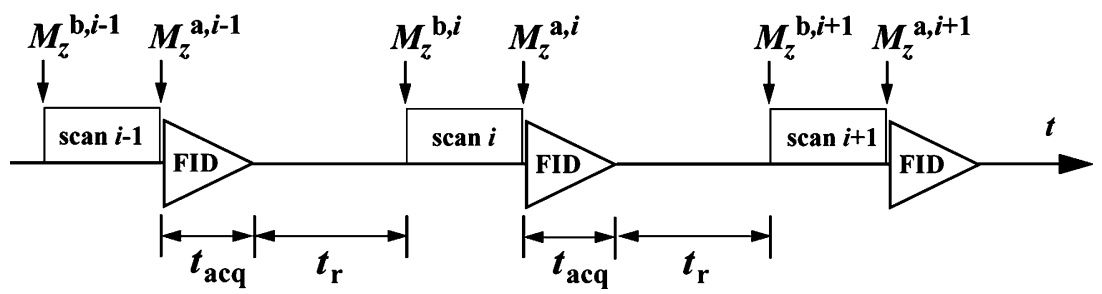

Figure 1. Definitions of the principal time points and time periods in the NMR experiments used in the present study. Indicated are three successive recordings ("scan") using a given experimental scheme (see Materials and Methods) and the associated free induction decay (FID), the acquisition and interscan periods, $t_{\text {acq }}$ and $t_{\mathrm{r}}$, and the time points where the water polarizations $b$ efore and $a$ fter the pulse sequence of a scan, $M_{z}^{\mathrm{b}}$ and $M_{z}^{\mathrm{a}}$, are measured (see text).

water magnetization, $f$-values $\lesssim 0.1$ are common. In heteronuclear $\left[{ }^{15} \mathrm{~N},{ }^{1} \mathrm{H}\right]$-correlation experiments carefully designed to maintain the water polarization, $f$-values between 0.7 and 0.95 can be obtained.

During the acquisition time, $t_{\text {acq, }}$ and the interscan delay, $t_{\mathrm{r}}$, the polarization $M_{z}^{\mathrm{a}, i}$ relaxes exponentially towards $M_{z}^{\mathrm{b}, i+1}$ (Figure 1),

$$
M_{z}^{\mathrm{b}, i+1}=M_{0}-\left(M_{0}-M_{z}^{\mathrm{a}, i}\right) \cdot \exp \left(-\frac{t}{T_{1}}\right)
$$

where $M_{0}$ is the equilibrium water polarization, $t=t_{\text {acq }}+t_{\mathrm{r}}$, and $T_{1}$ is the longitudinal relaxation time of the water protons (Bloch, 1946). With the Equations 1 and $2, M_{z}^{\mathrm{a}, i}$ and $M_{z}^{\mathrm{b}, i}$ can be calculated iteratively for all values of $i$, based on knowledge of the values for $f, T_{1}$ and the starting value $M_{z}^{\mathrm{b}, 1} . f$ and $T_{1}$ can be determined experimentally, as described in the Material and Methods section. Usually an experiment starts with equilibrium polarization of water, $M_{z}^{\mathrm{b}, 1}=M_{0}$. Once the experiment is started, the water polarization before the rf-pulses approaches a steady-state value, $M_{z}^{\mathrm{b}, \mathrm{ss}}=\lim _{i \rightarrow \infty} M_{z}^{\mathrm{b}, i}$, and correspondingly, $M_{z}^{\mathrm{a}, \mathrm{ss}}=\lim _{i \rightarrow \infty} M_{z}^{\mathrm{a}, i}$. Using Equations 1 and 2 yields

$$
M_{z}^{\mathrm{a}, \mathrm{ss}}=f \cdot M_{z}^{\mathrm{b}, \mathrm{ss}}=f \cdot \frac{1-\exp \left(-t / T_{1}\right)}{1-f \cdot \exp \left(-t / T_{1}\right)} \cdot M_{0}
$$

Equation 3 enables the calculation of the steadystate water polarization for a given NMR experiment from the values of $f$ and $T_{1}$, which can both be measured separately for a given experimental setup (see Material and Methods). It is convenient to use the normalized magnetizations $M_{z}^{\mathrm{a}, i} / M_{0}$ and $M_{z}^{\mathrm{b}, i} / M_{0}$ in the calculations, which directly indicate the percentage of preserved water polarization.

\section{Relaxation induced by paramagnetic agents}

The influence of paramagnetic chelated gadolinium ions on the nuclear longitudinal and transverse spin relaxation times can be described (Lauffer, 1987; Caravan et al., 1999) by

$$
\frac{1}{T_{i}}=\frac{1}{T_{i}^{\mathrm{d}}}+r_{i} \cdot[A], \quad i=1,2
$$

where $T_{i}$ stands for the longitudinal and transverse relaxation times, $T_{1}$ and $T_{2}, r_{1}$ and $r_{2}$ are the longitudinal and transverse relaxivities due to the paramagnetic reagent, and $[A]$ is the concentration of the relaxation agent. $T_{1}^{\mathrm{d}}$ and $T_{2}^{\mathrm{d}}$ are the "diamagnetic" longitudinal and transverse relaxation times, respectively, in the absence of a relaxation agent. A recent detailed discussion of the relaxation mechanisms described by Equation 4 can be found, for example, in (Caravan et al., 1999).

When working with protein solutions, Equation 4 applies to the relaxation of nuclear spins in the protein as well as the solvent water protons, albeit with different values for $r_{i}$. Taking Gd(DOTA) ${ }^{-}$(Chang et al., 1993) as an example, which is a typical relaxation agent used in magnetic resonance imaging (MRI), eight of the nine coordination sites of the gadolinium ion are occupied by the chelating molecule DOTA ${ }^{4-}$. One water molecule at a time can bind to the remaining ninth coordination site, where it can approach close to the gadolinium ion. In contrast, close contact with the protein surface is prevented by the chelating ligand. This is the basis for the pronouncedly selective relaxation enhancement on the water protons, since paramagnetic relaxation by $\mathrm{Gd}^{3+}$ is dominated by dipolar coupling and hence dependent on the inverse sixth power of the 
distance between the nuclear spin considered and the electronic spin.

\section{Materials and methods}

\section{Sample preparation}

The protein GroEL is a chaperonin from E. coli consisting of 14 subunits, which have identical conformations and thus give rise to identical NMR signals. Uniformly ${ }^{15} \mathrm{~N}$ - and ${ }^{2} \mathrm{H}$-labelled GroEL was expressed and purified as described earlier (Fiaux et al., 2002). After purification, the aqueous protein solution was adjusted to $25 \mathrm{mM}$ potassium phosphate at $\mathrm{pH} 6.2,20 \mathrm{mM} \mathrm{KCl}$ and $10 \% \mathrm{D}_{2} \mathrm{O}$. The final concentration of GroEL was $0.14 \mathrm{mM}$, corresponding to $2 \mathrm{mM}$ in monomers.

The tetrapeptide $\mathrm{H}-\mathrm{Gly}-\mathrm{Gly}-\mathrm{Glu}-\mathrm{Ala}-\mathrm{OH}$ (GGEA) was purchased from BACHEM AG (Switzerland) and used without further purification. Lyophilized GGEA was dissolved to a final concentration of 1 in $50 \mathrm{mM}$ potassium phosphate buffer at $\mathrm{pH} 4.2$ containing $10 \% \mathrm{D}_{2} \mathrm{O}$.

\section{Titration with paramagnetic reagents}

Highly concentrated solutions of paramagnetic reagents were used for the titrations. For example, stock solutions of 50,100 and $500 \mathrm{mM} \mathrm{Gd}$ (DOTA) ${ }^{-}$were added to the protein solution to achieve $0.5,1 \mathrm{mM}$, or larger concentration steps. Dilution effects have been neglected in the data analysis, since the change in volume per titration step never exceeded $1 \%$, and at most four titration steps were included in any given experiment. After the measurements, Gd(DOTA) ${ }^{-}$could be removed from the GroEL sample by a buffer exchange, using Amicon Ultra filters (Millipore) with the appropriate molecular weight cutoff.

\section{NMR experiments}

With the tetrapeptide GGEA, 1D ${ }^{1} \mathrm{H}-\mathrm{NMR}$ experiments were recorded using the pulse Scheme (5), which includes a 3-9-19 WATERGATE pulse train for water suppression (Sklenar et al., 1993).

$$
90^{\circ} \text {-WATERGATE-acq. }
$$

The interscan delay was $1 \mathrm{~s}$, and 64 transients were accumulated. $16 \mathrm{k}$ complex points were recorded during the acquisition time of $t_{\text {acq }}=780 \mathrm{~ms}$, and the FID was multiplied with a cosine window function and zero-filled to $32 \mathrm{k}$ points prior to Fourier transformation. No baseline correction was applied.

$2 \mathrm{D}\left[{ }^{15} \mathrm{~N},{ }^{1} \mathrm{H}\right]$-CRIPT-TROSY experiments were recorded with the pulse Scheme (6) (Riek et al., 1999; Riek et al., 2002),

$$
\begin{aligned}
& 90\left({ }^{1} \mathrm{H}\right)-\mathrm{T} / 2-180\left({ }^{1} \mathrm{H}\right)-\mathrm{T} / 2-90\left({ }^{1} \mathrm{H}\right) \\
& -90\left({ }^{15} \mathrm{~N}\right)-\mathrm{t}_{1}-90\left({ }^{15} \mathrm{~N}\right)-90\left({ }^{1} \mathrm{H}\right)-\text { acq. }
\end{aligned}
$$

where the water magnetization was flipped back by the application of a selective $90{ }^{\circ}\left({ }^{1} \mathrm{H}\right)$ pulse on the water resonance with each non-selective $90^{\circ}\left({ }^{1} \mathrm{H}\right)$ pulse (for details, including the phase cycling used, see Figure SF1 in Supplementary Material). From a buildup experiment (Riek et al., 2002), the optimal heteronuclear magnetization transfer time $\mathrm{T}$ for the $800 \mathrm{kDa}$ GroEL was determined to be $1.4 \mathrm{~ms}$, and the optimal interscan delay was determined to be $300 \mathrm{~ms} .1024$ complex points were recorded during an acquisition time of $97 \mathrm{~ms}$, and prior to Fourier transformation the FID was multiplied with a $20^{\circ}$ shifted sine bell and zero-filled to 2048 complex points. In the ${ }^{15} \mathrm{~N}$ dimension, 100 complex points were measured, with a maximal evolution time of $22 \mathrm{~ms}$, and the data in $t_{1}$ were multiplied with an exponential window function with a line broadening factor of $20 \mathrm{~Hz}$ and zero-filled to 256 complex points before Fourier transformation. The baseline was corrected using the IFLAT method (Bartels et al., 1995a) in the $\omega_{2}\left({ }^{1} \mathrm{H}\right)$-dimension and polynomials in the $\omega_{1}\left({ }^{15} \mathrm{~N}\right)$-dimension. To obtain a $2 \mathrm{D}$ $\left[{ }^{15} \mathrm{~N},{ }^{1} \mathrm{H}\right]$-CRIPT-TROSY spectrum with saturation of the water resonance, the second water flipback pulse in the pulse sequence was removed, resulting in dephasing of the water signal by the subsequent strong magnetic field gradient $\mathrm{G}_{2}$ (Figure SF1).

The pulse Scheme (7) presents the test NMR experiment used to verify the model calculations performed with the use of Equations 1-4.

$$
90_{-\mathrm{x}}^{\circ}\left(\mathrm{H}_{2} \mathrm{O}\right)-90_{\mathrm{x}}\left({ }^{1} \mathrm{H}\right)-\mathrm{G}_{\mathrm{z}}-\mathrm{acq} \text {. }
$$

It consists of a selective excitation pulse with phase $-x$ on the water resonance, followed by a hard pulse on protons with phase $\mathrm{x}$, and a strong 
magnetic field gradient along the $+z$ axis. By variation of the properties of the selective pulse, the flip-back ratio $f$ can readily be adjusted. In the present work the selective pulse had a Gaussian shape and a duration of $1.0 \mathrm{~ms}$.

The reference water polarization was measured with the pulse Scheme (8),

Tap pulse-acq.,

where the water magnetization along the $+z$ axis (the "water polarization") is tilted slightly towards the transverse plane by a very short rectangular pulse with high radio-frequency power ("tap pulse"). With the resulting small tilt angles of typically $2^{\circ}$, radiation damping does not occur and the integral over the water signal recorded with Scheme (8) is proportional to the water polarization before the tap pulse. The small flip angle also reduces the bias arising from interscan delays that are too short to allow a complete relaxation to thermal equilibrium. The equilibrium polarization of the water, $M_{0}$, which is needed as a reference for the determination of the flip-back ratio $f$, was measured by applying pulse Scheme (8) to the system at thermal equilibrium. For measurements of the water polarization at a given time point during a NMR pulse scheme, the routine of pulse Scheme (8) was supplemented with a strong magnetic field gradient along the $z$-axis prior to the tap pulse; in this way all transverse magnetization already present before the tap pulse, which could otherwise lead to spurious signals, was dephased. The water polarizations $M_{z}^{\mathrm{b}, i}$ and $M_{z}^{\mathrm{a}, i}$ (Figure 1) were thus measured by truncating the pulse Schemes (5), (6) and (7) at the appropriate time points, whereupon the water polarization was measured with a tap pulse preceded by a $z$-gradient pulse.

The longitudinal ${ }^{1} \mathrm{H}$ relaxation time of water, $T_{1}$, was measured using standard inversion recovery experiments (Ernst et al., 1987), for which the receiver coil was detuned to avoid radiation damping.

All NMR spectra were recorded on a Bruker DRX 750 spectrometer at $25{ }^{\circ} \mathrm{C}$ using a ${ }^{1} \mathrm{H}$ $\left\{{ }^{15} \mathrm{~N},{ }^{13} \mathrm{C}\right\}$-triple resonance probehead equipped with a shielded $z$-gradient coil. The data were processed with the programs XWINNMR and PROSA (Güntert et al., 1992), and analyzed with XWINNMR and XEASY (Bartels et al., 1995b).

\section{Results and discussion}

Water polarization in multiple-scan NMR experiments with $\mathrm{H}_{2} \mathrm{O}$ and with paramagnetic salt solutions

As discussed in the introduction, the NMR signal intensity of large proteins is directly coupled to the extent of the steady-state water polarization. It is therefore of interest to maximize the water polarization during the entire experiment. Here, we apply the formalism of Equations 1-3 to describe the propagation of the water polarization, and compare the results of these model calculations with measurements using the NMR pulse Scheme (7). With regard to the water flip-back behavior, this simple pulse sequence mimics the NMR experiments that are typically used for studies of macromolecules. For the examples shown in Figure 2, the experimental data fit very closely with the model predictions, and the same was observed with several other measurements (data not shown). The calculations were based on experimental values for $T_{1}$ and $f$ which had been determined in separate experiments, as described in Materials and methods, and no adjustable parameter was used to match theory and experiment. The close agreement between experimental and calculated data supports the assumption made in Equation 1, that to a good approximation the quantity $f$ adopts a constant value for multi-scan NMR experiments designed to preserve the water polarization.

The data of Figure 2 represent typical situations encountered in biomolecular NMR, except that the $T_{1}$ value for the water may be somewhat shorter in protein solutions than in pure $\mathrm{H}_{2} \mathrm{O}$. Figure 2a shows the magnitude of the water polarization in a conventional experiment with water flip-back pulses. The steady-state water polarization during this experiment depends strongly on the interscan delay, $t_{\mathrm{r}}$, and the water flip-back ratio, $f$. But even with $f=90 \%$, a desirably short interscan delay $t_{\mathrm{r}}=600 \mathrm{~ms}$, as chosen here, results in a steady-state polarization for pure water of only $0.6 M_{0}$ (with identical conditions, Equation 3 predicts only slightly higher steady-state polarization in protein solutions). Figure $2 \mathrm{a}$ also shows how the approach of the water polarization to its steady-state depends on the value chosen for $t_{\mathrm{r}}$, and this figure can thus serve as a guideline for proper 

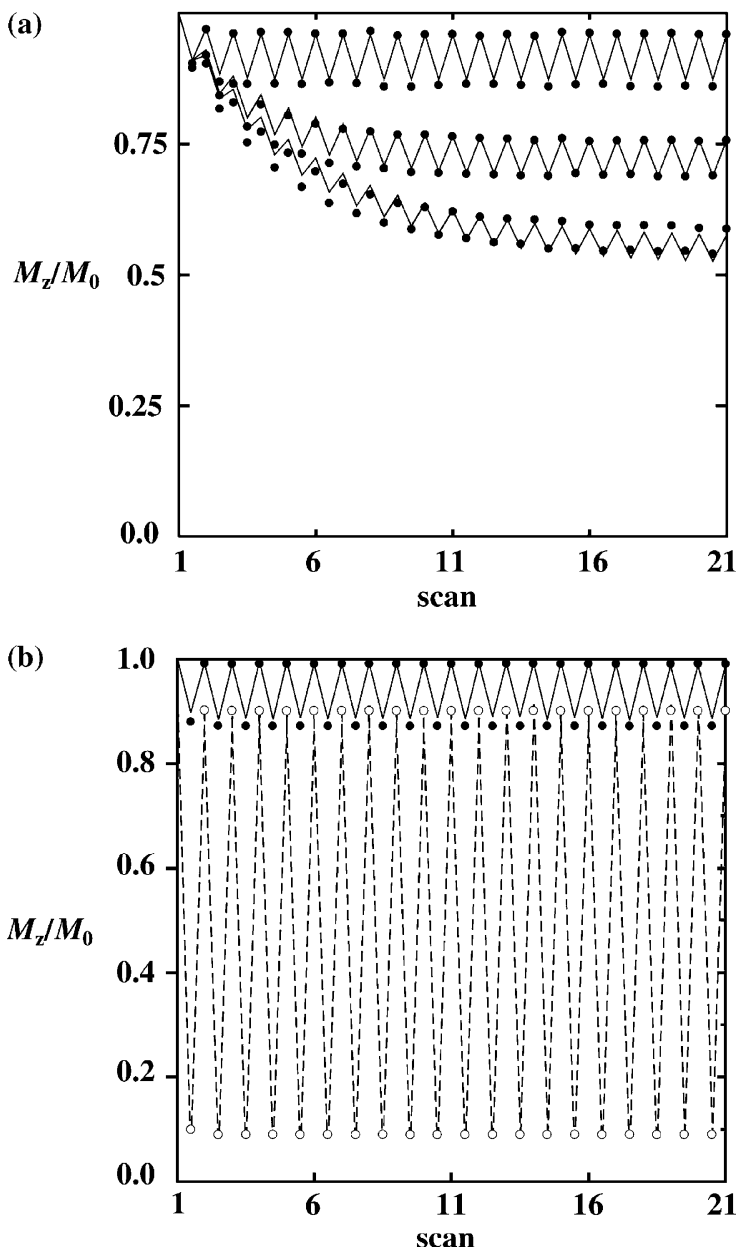

selection of the number of dummy scans at the start of an experiment.

With the addition of a paramagnetic relaxation agent, the solvent water $T_{1}$ values can be greatly reduced. For example, the $T_{1}$-values of water measured in $1 \mathrm{mM}$ solutions of the tetrapeptide GGEA containing 0,1 , and $2 \mathrm{mM} \mathrm{Gd(DOTA)}{ }^{-}$are 3500, 310, and $160 \mathrm{~ms}$ (Figure 3). From these data, Equation 4 yields a relaxivity of $r_{1}=3.4 \mathrm{~Hz} / \mathrm{mM}$ for Gd(DOTA) ${ }^{-}$acting on the bulk water protons. Figure $2 b$ shows the evolution of the water polarization in multiple-scan NMR experiments with a $2 \mathrm{mM} \mathrm{Gd(DOTA)}{ }^{-}$solution. With a flip-back rate of $f=90 \%$ and an interscan delay of only $300 \mathrm{~ms}$, the steady-state water polarization, $M_{z}^{\mathrm{b}, \mathrm{ss}}$, is virtually identical to the equilibrium polarization $M_{0}$

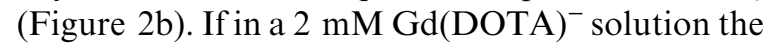
water polarization was completely destroyed during the pulse sequence, a value for $M_{z}^{\mathrm{b}, \text { ss }}$ of about
Figure 2. Time evolution of the water polarization, $M_{z} / M_{0}$, in a multiple-scan NMR experiment. Filled and open circles represent experimental data, solid and broken lines represent the results of model calculations based on the Equations 1 and 2. For each recording, the water polarizations before and after the scan $\left(M_{z}^{\mathrm{b}, i}\right.$ and $M_{z}^{\mathrm{a}, i}$ in Figure 1) are plotted, starting with $M_{z}^{\mathrm{b}, 1}$. The equilibrium polarization of the water, $M_{0}$, was independently measured (see Materials and Methods). Theoretical values were calculated iteratively, using experimentally determined longitudinal relaxation times $T_{1}$ and flip-back ratios $f$, which had been measured as described in Materials and Methods. (a) Data for pure $\mathrm{H}_{2} \mathrm{O}$, which has a $T_{1}$-value of $4500 \mathrm{~ms}$. Model calculations and experiments were based on a water flip-back ratio of $f=90 \%$. Three data sets are shown which used, from top to bottom, interscan delays of $t_{\mathrm{r}}=5.0 \mathrm{~s}$, $1.0 \mathrm{~s}$, and $0.6 \mathrm{~s}$, respectively. Experiment (7) was used with an acquisition time of $t_{\text {acq }}=97 \mathrm{~ms}$. (b) Data for a $2 \mathrm{mM}$ aqueous $\mathrm{Gd}(\mathrm{DOTA})^{-}$solution, which has a $T_{1}$-value of $180 \mathrm{~ms}$ for the water protons. Solid lines represent the model prediction for a water flip-back ratio of $f=90 \%$, and filled circles show the result of a corresponding experiment with pulse Scheme (7). Broken lines and open circles show predictions and experimental results obtained with a water flip-back ratio of $f=10 \%$ in the experimental setup of pulse Scheme (7); thereby, the water resonance is saturated at the beginning of each scan with a selective rf-pulse on the water resonance followed by a strong magnetic field gradient pulse. For both values of $f$ the acquisition time was $97 \mathrm{~ms}$ and the interscan delay was $300 \mathrm{~ms}$.

$0.9 M_{0}$ was achieved (Figure $2 \mathrm{~b}$ ), which contrasts with $M_{z}^{\mathrm{b}, \mathrm{ss}} \approx 0.09 M_{0}$ for pure water.

\section{Selective solvent water relaxation enhancement in protein solutions by $\mathrm{Gd}(\mathrm{DOTA})^{-}$}

For the purpose of this study, we looked for a paramagnetic relaxation agent which would strongly reduce the $T_{1}$-value of the water protons without significantly affecting the protein resonances. Such relaxation agents must allow close access of water molecules to their unpaired electrons while keeping the protein protons at a comparatively long distance. This requirement is well met by the gadolinium ion complex Gd (DOTA) ${ }^{-}$, which has one free metal coordination site that is readily accessible for water but cannot, for steric reasons, be accessed closely by protein protons. Figure 3 surveys the effects of variable concentrations of $\mathrm{Gd}(\mathrm{DOTA})^{-}$and free $\mathrm{Gd}^{3+}$ ions, respectively, on the water proton $T_{1}$-values and the line widths of the peptide in solutions of the tetrapeptide GGEA. The key observation is that with Gd(DOTA) ${ }^{-}$the water $T_{1}$-value can be reduced 10 fold without significant line broadening of the peptide ${ }^{1} \mathrm{H}$-NMR lines, whereas with $\mathrm{Gd}^{3+}$ a $20 \%$ reduction of the water $T_{1}$-value is paralleled by 


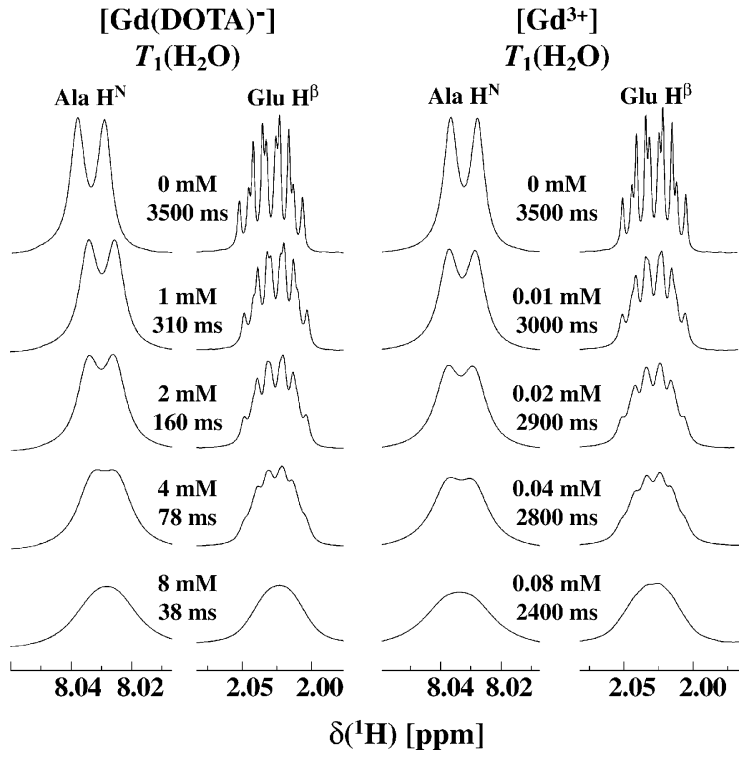

Figure 3. Effects of $\mathrm{Gd}(\mathrm{DOTA})^{-}$and $\mathrm{Gd}^{3+}$ on the $1 \mathrm{D}{ }^{1} \mathrm{H}-$ NMR spectrum of $\mathrm{H}-\mathrm{Gly}-\mathrm{Gly}-\mathrm{Glu}-\mathrm{Ala}-\mathrm{OH}$ and on the $T_{1^{-}}$ value of the water in a $1 \mathrm{mM}$ solution of the peptide in $50 \mathrm{mM}$ aqueous phosphate buffer containing $10 \% \mathrm{D}_{2} \mathrm{O}, \mathrm{pH}=4.2$. Pulse Scheme (5) was used. The two columns on the left show the effects of stepwise addition of $\mathrm{Gd}(\mathrm{DOTA})^{-}$on the resonances of the amide proton of Ala 4 and the $\beta$-protons of Glu3. The two columns on the right show the effect of $\mathrm{GdCl}_{3}$ on the same resonances. The concentrations of $\mathrm{Gd}(\mathrm{DOTA})^{-}$and $\mathrm{Gd}^{3+}$, respectively, were selected in such a way that the effective $T_{2^{-}}$values of the peptide resonances were approximately the same for both paramagnetic relaxation agents. The concentrations of the relaxation agents and the corresponding $T_{1}$-value of the water protons are indicated for each titration step.

nearly complete loss of the peptide ${ }^{1} \mathrm{H}$-NMR fine structure due to line broadening. In other words, closely similar effective $T_{2}$-values for the peptide ${ }^{1} \mathrm{H}$-NMR lines are obtained with 100 -fold higher concentration of $\mathrm{Gd}(\mathrm{DOTA})^{-}$than of $\mathrm{Gd}^{3+}$.

The relaxivity $r_{2}$ of $\operatorname{Gd}(\mathrm{DOTA})^{-}$on protein resonances is of the order $2-5 \mathrm{~Hz} / \mathrm{mM}$, as measured with the tetrapeptide GGEA (Figure 3) and with GroEL (Table 1). For nuclei that are not solvent-exposed, $r_{2}$ is even smaller. Although the resulting line broadening is clearly visible for small peptides with narrow natural ${ }^{1} \mathrm{H}-\mathrm{NMR}$ line widths

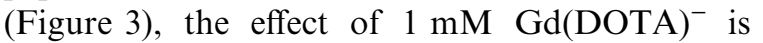
hardly noticeable for large proteins with very broad ${ }^{1} \mathrm{H}-\mathrm{NMR}$ lines. For example, in GroEL the natural ${ }^{1} \mathrm{H}$-NMR linewidths are of the order of $50-80 \mathrm{~Hz}$ (Table 1), so that an increase of $2-5 \mathrm{~Hz}$ due to the presence of $1 \mathrm{mM} \mathrm{Gd(DOTA)})^{-}$is for most practical purposes acceptable. Overall, these observations show that $\operatorname{Gd}(\text { DOTA })^{-}$is a suitable
Table 1. ${ }^{1} \mathrm{H}$ linewidths of six resolved cross-peaks in four $2 \mathrm{D}$ $\left[{ }^{15} \mathrm{~N},{ }^{1} \mathrm{H}\right]$-CRIPT-TROSY spectra of GroEL recorded with variable $\mathrm{Gd}(\mathrm{DOTA})^{-}$concentrations and otherwise identical conditions, as described in Figure 5

\begin{tabular}{|c|c|c|c|c|c|c|}
\hline \multirow[t]{2}{*}[\mathrm{Gd}(\mathrm{DOTA})^{-}]{} & \multicolumn{6}{|c|}{ Linewidth $[\mathrm{Hz}]^{\mathrm{a}}$} \\
\hline & I & II & III & IV & V & VI \\
\hline $0 \mathrm{mM}$ & 55 & 53 & 83 & 60 & 57 & 55 \\
\hline $0.5 \mathrm{mM}$ & 64 & 57 & 84 & 65 & 57 & 59 \\
\hline $1 \mathrm{mM}$ & 63 & 59 & 83 & 64 & 62 & 61 \\
\hline $2 \mathrm{mM}$ & 65 & 60 & 86 & 64 & 60 & 66 \\
\hline
\end{tabular}

${ }^{\mathrm{a}}$ Full line width at half height as determined by linear interpolation between data points along $\omega_{2}\left({ }^{1} \mathrm{H}\right)$. The digital resolution of the spectra was $5 \mathrm{~Hz} /$ point along $\omega_{2}\left({ }^{1} \mathrm{H}\right)$ and $17 \mathrm{~Hz} /$ point along $\omega_{1}\left({ }^{15} \mathrm{~N}\right)$. The peaks I-VI are identified in Figure 5a.

agent for managing the solvent water polarization in biomacromolecular NMR without deteriorating the NMR signals of the solute.

\section{Practical applications}

The addition of $\operatorname{Gd}(\mathrm{DOTA})^{-}$can be used to enhance the sensitivity for the recording of NMR spectra of large molecular or supramolecular structures by increasing the steady-state water polarization in experiments with water flip-back pulses. In 2D $\left[{ }^{15} \mathrm{~N},{ }^{1} \mathrm{H}\right]$-CRIPT-TROSY experiments with the $800 \mathrm{kDa}$ chaperone protein GroEL, the steady-state polarization of the water could be increased from $0.68 M_{0}$, which was obtained with optimized water flip-back pulses in the absence of a paramagnetic reagent, to $0.97 M_{0}$

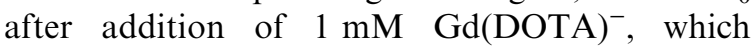
resulted in a nearly 1.3-fold gain of the protein signal intensity (Table 2). This result was obtained from an analysis of the peak heights of 71 resolved

Table 2. Survey of the influence of the addition of variable concentrations of Gd(DOTA) ${ }^{-}$on the $2 \mathrm{D}\left[{ }^{15} \mathrm{~N},{ }^{1} \mathrm{H}\right]$-CRIPTTROSY spectrum of GroEL ${ }^{a}$

\begin{tabular}{lcll}
\hline$\left[\mathrm{Gd}(\mathrm{DOTA})^{-}\right][\mathrm{mM}]$ & $T_{1}\left(\mathrm{H}_{2} \mathrm{O}\right)[\mathrm{ms}]$ & $M_{z}^{\mathrm{b}, \mathrm{ss}}[\%]^{b}$ & $\mathrm{I}_{\text {rel }}^{\mathrm{c}}$ \\
\hline 0.0 & 3500 & 68 & 1.00 \\
0.5 & 580 & 93 & 1.27 \\
1.0 & 310 & 97 & 1.28 \\
2.0 & 160 & 98 & 1.23 \\
\hline
\end{tabular}

${ }^{\mathrm{a}}$ For experimental details see Figure 5.

${ }^{\mathrm{b}} M_{z}^{\mathrm{b}, \mathrm{ss}}$ is expressed in \% of $M_{0}$.

${ }^{c}$ Average relative peak height of 71 resolved resonances (see text and supplementary material). 


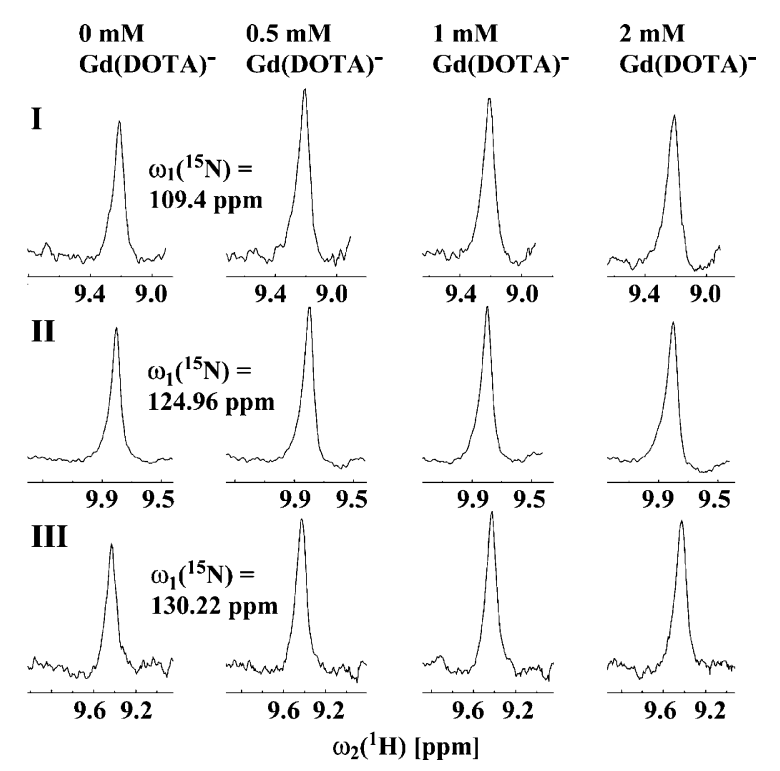

Figure 4. Cross-sections from four $2 \mathrm{D}\left[{ }^{15} \mathrm{~N},{ }^{1} \mathrm{H}\right]$-CRIPT-TROSY spectra of GroEL recorded at various concentrations of $\operatorname{Gd}(\text { DOTA })^{-}$, as given at the top of each column. Each row shows the same resonance from the four spectra, with its spectral positions indicated. The three resonances I-III are marked in Figure 5a.

resonances in these spectra (Figures 4 and 5; Table ST1 in Supplementary Material). Of the 547 residues in GroEL, only about $20 \%$ give rise to observable cross-peaks in a $2 \mathrm{D}\left[{ }^{15} \mathrm{~N},{ }^{1} \mathrm{H}\right]$-CRIPTTROSY experiment (Riek et al., 2002). Among the observable resonances, 71 were found to be well resolved and thus suitable for the present analysis. The following observations show that at least part of these resonances originate from structured parts of the protein, including regular secondary structures: the large chemical shift dispersion (Table ST1), the findings that a transfer time $\mathrm{T}$ of $1.4 \mathrm{~ms}$ was optimal for the $2 \mathrm{D}\left[{ }^{15} \mathrm{~N},{ }^{1} \mathrm{H}\right]-$ CRIPT-TROSY experiments (Pulse Scheme (6), Figure SF1), and the line widths of $40-100 \mathrm{~Hz}$ in the $\omega_{2}\left({ }^{1} \mathrm{H}\right)$ dimension. The regular secondary structure elements are mostly buried in the interior of the molecule (Xu et al., 1997), indicating that the present experiments yielded improvements of signals originating from the protein core. If some of the observed nuclei were located close to the protein surface, their transverse relaxation rates would be more strongly accelerated by the paramagnetic ions than for nuclei in the interior of the molecule. The resulting broadening of the resonance lines would then decrease the apparent signal enhancement measured as the change in peak heights. Since the data in Table 2 possibly include measurements for surface-exposed ${ }^{15} \mathrm{~N}-{ }^{1} \mathrm{H}$ moieties, they represent a lower limit of the actual gain in sensitivity. Increasing the $\operatorname{Gd}(\text { DOTA })^{-}$ concentration beyond $1 \mathrm{mM}$ does not result in further gain for the protein signals, since the small additional increase in the water polarization (Table 2) is overcompensated by the increased transverse relaxation rates of the protein resonances (Table 1). Paramagnetic relaxation agents were previously used to obtain signal enhancement in proteins through direct dipolar relaxation enhancement of the protein nuclei of interest (Eletsky et al., 2003); this approach is intrinsically limited by the concomitant broadening of the protein resonances.

A second application of paramagnetic relaxation agents is based on the experiment in Figure $2 \mathrm{~b}$, which suggests that water flip-back pulses might be omitted altogether. This could be of interest whenever water flip-back segments are difficult to adjust, or if they cause substantial signal loss for large molecules because they prolong the pulse sequence. The concern here is not limited to the loss of water polarization, but it also considers that residual transverse water magnetization may interfere with the signal acquisition. If the requirements of a given pulse sequence do not permit the use of appropriate water suppression elements, the remaining strong water signal may lead to a receiver overflow and the recording of a digitized signal can become difficult or impossible. As an illustration, Figure 5 compares two 2D $\left[{ }^{15} \mathrm{~N},{ }^{1} \mathrm{H}\right]$-CRIPT-TROSY spectra of GroEL, which were measured, respectively, in the absence of $\operatorname{Gd}(\text { DOTA })^{-}$using carefully optimized water flip-back pulses, or in the presence of $2 \mathrm{mM}$ Gd(DOTA) ${ }^{-}$with complete destruction of the water polarization during the pulse sequence. The $T_{1}$-value of water was $170 \mathrm{~ms}$ in a solution with $2 \mathrm{mM}$ Gd(DOTA) ${ }^{-}$(Table 2), and about $90 \%$ of the equilibrium water polarization was recovered during $400 \mathrm{~ms}$ of acquisition and interscan periods (Figure 2b). The spectral quality in Figure $5 \mathrm{~b}$ is markedly improved when compared to Figure $5 \mathrm{a}$, and the cross-sections $5 \mathrm{a}^{\prime}$ to $5 \mathrm{~b}^{\prime \prime}$ clearly show that this improvement was achieved without loss of sensitivity for detection of the signals of interest, which have comparable signalto-noise ratios in the two experiments of Figure 5, 

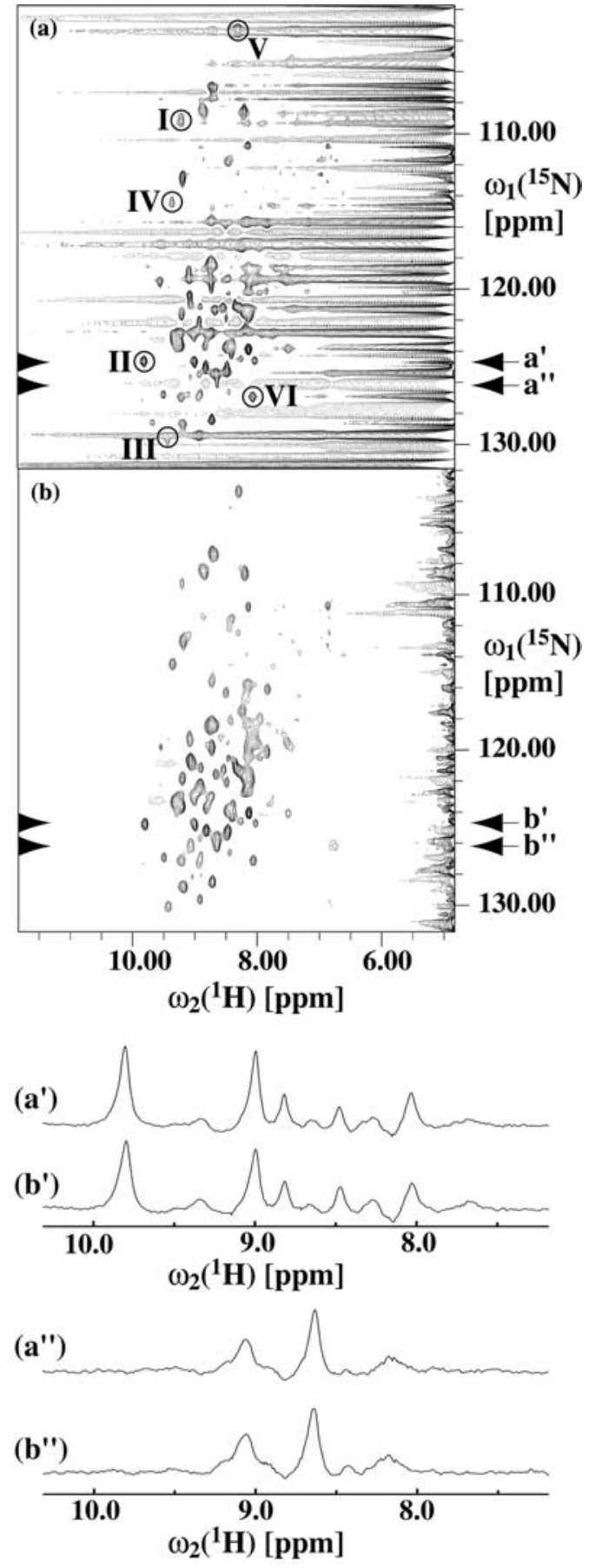

$\mathrm{a}$ and $\mathrm{b}$ (a comparable water suppression could be obtained without addition of Gd(DOTA) ${ }^{-}$, but the protein signals would be drastically reduced as well (Akasaka et al., 1978; Stoesz et al., 1978)).

In conclusion, this paper presents a novel use of selective relaxation agents to enhance the NMR
Figure 5. 2D $\left[{ }^{15} \mathrm{~N},{ }^{1} \mathrm{H}\right]$-CRIPT-TROSY spectra (Riek et al., 1999; 2002) of an aqueous solution of uniformly ${ }^{15} \mathrm{~N}$ - and ${ }^{2} \mathrm{H}$ labeled GroEL (protein concentration $0.14 \mathrm{mM}, 20 \mathrm{mM} \mathrm{KCl}$, $25 \mathrm{mM}$ phosphate at $\mathrm{pH}=6.2,10 \% \mathrm{D}_{2} \mathrm{O}$ ) recorded with pulse Scheme (6) (for details see Materials and Methods and Figure SF1). (a) Spectrum recorded without addition of Gd(DOTA) ${ }^{-}$, using optimized water flip-back pulses. Roman numerals and circles indicate peaks that are analyzed in Figure 4 and Table 1.

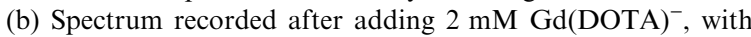
saturation of the water resonance using a strong magnetic field gradient in the pulse sequence. Except for the different water handling, (a) and (b) were recorded under identical conditions, as described in Materials and Methods and Figure SF1. Both spectra were plotted with the same contour levels, and no baseline correction was applied. $\left(a^{\prime}\right),\left(b^{\prime}\right),\left(a^{\prime \prime}\right)$ and $\left(b^{\prime \prime}\right)$ Crosssections from (a) and (b) taken at the positions indicated by the arrows. To enable a reliable comparison of the signal intensities, a baseline correction was applied to the cross-sections.

spectral quality for large proteins. The proposed procedures might be beneficial in all NMR experiments with large macromolecular systems where water flip-back pulses are routinely used. In addition to $\operatorname{Gd}(\text { DOTA })^{-}$used in this study, a wide range of metal chelates with different chemical properties are used as contrast agents in magnetic resonance imaging (MRI) (Caravan et al., 1999), and several of these reagents have already proven their usefulness in biomolecular NMR for mapping of protein surfaces (Liepinsh and Otting, 1997; Pintacuda and Otting, 2002; Hilty et al., 2004) or for resolving spectral overlap (Sattler and Fesik, 1997). Even higher selectivity of the relaxation effects on the solvent than with Gd(DOTA) ${ }^{-}$ may be found in future studies using different paramagnetic relaxation agents, to further minimize the relaxation effects on the protein resonances and thus to provide even larger signal enhancements, which might in turn make the presently proposed approach attractive also for smaller molecular weights.

Supplementary material to this paper is available in electronic format at http://dx.doi.org/10.1007/ s10858-005-3070-8.

\section{Acknowledgement}

We thank Prof. P. Bösiger (University of Zürich) for a gift of Gd(DOTA) ${ }^{-}$, and the ETH Zürich and the Schweizerischer Nationalfonds for financial support through the National Center for Competence in Research (NCCR) 'Structural Biology'. 


\section{References}

Akasaka, K., Konrad, M. and Goody, R.S. (1978) FEBS Lett., 96, 287-290.

Bartels, C., Güntert, P. and Wüthrich, K. (1995a) J. Magn. Reson. A, 117, 330-333.

Bartels, C., Xia, T.H., Billeter, M., Güntert, P. and Wüthrich, K. (1995b) J. Biomol. NMR, 6, 1-10.

Bax, A. and Grzesiek, S. (1993) Accounts Chem. Res., 26, 131-138.

Bloch, F. (1946) Phys. Rev., 70, 460-473.

Caravan, P., Ellison, J.J., McMurry, T.J. and Lauffer, R.B. (1999) Chem. Rev., 99, 2293-2352.

Chang, C.A., Francesconi, L.C., Malley, M.F., Kumar, K., Gougoutas, J.Z., Tweedle, M.F., Lee, D.W. and Wilson, L.J. (1993) Inorg. Chem., 32, 3501-3508.

Eletsky, A., Moreira, O., Kovacs, H. and Pervushin, K. (2003) J. Biomol. NMR, 26, 167-179.

Ernst, R.R., Bodenhausen, G. and Wokaun, A. (1987) Principles of Nuclear Magnetic Resonance in One and Two Dimensions, Oxford University Press, Oxford.

Fiaux, J., Bertelsen, E.B., Horwich, A.L. and Wüthrich, K. (2002) Nature, 418, 207-211.

Grzesiek, S. and Bax, A. (1993) J. Am. Chem. Soc., 115, 1259312594.

Güntert, P., Dötsch, V., Wider, G. and Wüthrich, K. (1992) J. Biomol. NMR, 2, 619-629.

Hilty, C., Wider, G., Fernández, C. and Wüthrich, K. (2004) Chembiochem, 5, 467-473.

Kay, L.E. (1997) Prog. Biophys. Mol. Biol., 63, 277-299.

Lauffer, R.B. (1987) Chem. Rev., 87, 901-927.

LeMaster, D.M. (1994) Prog. Nucl. Magn. Reson. Spectrosc., 26, 371-419.

Liepinsh, E. and Otting, G. (1997) Nat. Biotechnol., 15, 264-268.
Otting, G. (1997) Prog. Nucl. Magn. Reson. Spectrosc., 31, 259-285.

Pervushin, K., Riek, R., Wider, G. and Wüthrich, K. (1997) Proc. Natl. Acad. Sci. USA, 94, 12366-12371.

Pervushin, K., Vögeli, B. and Eletsky, A. (2002) J. Am. Chem. Soc., 124, 12898-12902.

Pintacuda, G. and Otting, G. (2002) J. Am. Chem. Soc., 124, 372-373.

Riek, R., Fiaux, J., Bertelsen, E.B., Horwich, A.L. and Wüthrich, K. (2002) J. Am. Chem. Soc., 124, 12144-12153.

Riek, R., Wider, G., Pervushin, K. and Wüthrich, K. (1999) Proc. Natl. Acad. Sci. USA, 96, 4918-4923.

Sattler, M. and Fesik, S.W. (1997) J. Am. Chem. Soc., 119, 7885-7886.

Sklenar, V., Piotto, M., Leppik, R. and Saudek, V. (1993) J. Magn. Reson. A, 102, 241-245.

Stoesz, J.D., Redfield, A.G. and Malinowski, D. (1978) FEBS Lett., 91, 320-324.

Wang, Y.X., Freedberg, D.I., Grzesiek, S., Torchia, D.A., Wingfield, P.T., Kaufman, J.D., Stahl, S.J., Chang, C.H. and Hodge, C.N. (1996) Biochemistry, 35, 12694-12704.

Wider, G. (1998) Prog. Nucl. Magn. Reson. Spectrosc., 32, $193-275$.

Wider, G., Hosur, R.V. and Wüthrich, K. (1983) J. Magn. Reson., 52, 130-135.

Wüthrich, K. (1986) NMR of Proteins and Nucleic Acids, Wiley, New York.

Wüthrich, K. (2003) Angew. Chem. Int. Ed., 42, 3340-3363.

Wüthrich, K. and Wider, G. (2003) Magn. Reson. Chem., 41, S80-S88.

Xu, Z.H., Horwich, A.L. and Sigler, P.B. (1997) Nature, 388, $741-750$. 